\title{
Percrete Placenta with Bladder Invasion: A Case Report and Literature Review
}

ISSN: 2640-9666

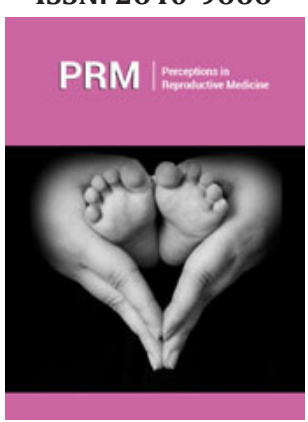

*Corresponding author: Malagón Reyes R, Surgeon General, Mexico

Submission: 㘹 July 17, 2020

Published: 僵 September 04, 2020

Volume 4 - Issue 1

How to cite this article: Malagón Reyes R, Soria Soriano EL, Garcia Gonzalez BN. Percrete Placenta with Bladder Invasion: A Case Report and Literature Review. Perception in Reproductive Medicine. 4(1). PRM. 000577. 2020.

DOI: 10.31031/PRM.2020.04.000577

Copyright@ Malagón Reyes R, This article is distributed under the terms of the Creative Commons Attribution 4.0 International License, which permits unrestricted use and redistribution provided that the original author and source are credited.

\author{
Malagón Reyes $\mathrm{R}^{1 *}$, Soria Soriano EL ${ }^{2}$ and Garcia Gonzalez $\mathrm{BN}^{3}$ \\ ${ }^{1}$ Surgeon General, Mexico \\ ${ }^{2}$ Resident Gynecology Doctor, Mexico \\ ${ }^{3}$ Gynecology Resident Physician, Mexico
}

\section{Summary}

Monica Pretelini Saenz Maternal Perinatal Hospital, Toluca, Mexico. We present a clinical case of a pregnant patient diagnosed with a percrete placenta at the Monica Pertelini Sáenz Maternal Perinatal Hospital. Antenatal diagnosis is described, as well as intraoperative management and technique. They make themselves known the immediate and mediate complications presented by the patient, in addition to the post-discharge follow-up. Finally, a review of the literature is carried out and recommendations for better multidisciplinary management are suggested.

\section{Abstract}

We presented a clinical case of a pregnant patient diagnosed with a percrete placenta at the Monica Pretelini Saenz Maternal Perinatal Hospital. Prenatal diagnosis is described, as well as intraoperative management and technique. The immediate and mediate complications presented by the patient are disclosed, as well as the follow-up after her progress. Finally, a review of the literature is carried out and recommendations are sought for better multidisciplinary management. Placental accretion is associated with maternal morbidity and mortality.

\section{Introduction}

Accreted Placenta Spectrum (AP) is the general term applied to abnormal adherence of the placental trophoblast to the uterine myometrium, also known as morbidly attached placenta. Placental accreta includes placenta accreta (fixation of the placenta to the myometrium without intervening (deci-dua), placenta increta (invasion of the trophoblast to the myometrium), and placenta percreta (PP) (invasion through the myometrium, serous, and surrounding structures) [1]; Figure 1. The first PA case reports were published in the literature in 1920, the first series in 1937 by obstetrician Frederick C. Irving and pathologist Arthur TH of the Boston Lying-In Hospital [2]. Risk factors are any procedure or manipulation that damages the endometrium, including uterine curettage, myomectomy, endometrial ablation, uterine artery embolization, or manual removal of the placenta [1]. The fetus and maternal complications are mainly the result of massive bleeding. In turn, this leads to disseminated intravascular coagulation, multiorgan failure, the need for additional surgery that includes hysterectomy, thromboembolism, and even death. The median estimated blood loss in cohorts of acrets ranges from 2,000 to 7,800 $\mathrm{mL}$ [3]. Ultrasound is recommended as a first-line imaging study and represents the gold standard for the diagnosis of placental accretion. The sensitivity and specificity of ultrasound in the 2nd and 3rd trimester is 80\%-90\% [4]. Doppler findings associated with PA include blood flow turbulent neo saccular, increased subplantar vascularization (Figure 2), vessels connecting the placenta to the margin. Magnetic resonance imaging has a sensitivity and specificity of $80 \%-90 \%$, it is recommended to be performed only when the a priori risk is high due to its high cost and it has been found to be useful in cases of posterior placenta previa, obesity morbid or in cases of potential invasion to bladder 
[1]; Figure 3. There is an apparent invasion of the rectus abdominis muscles. The hyperintense line that separates the muscles of the uterus is lost. One of the most frequent complications in this pathology is massive hemorrhage and massive transfusion with its consequent complications and a third presents incidental injury to the bladder. Ureteral injury, vesicovaginal fistula, and surgical reoperation are complications that occur less frequently. count. (1) Finally, maternal death has been reported in up to $7 \%$ of cases [3].
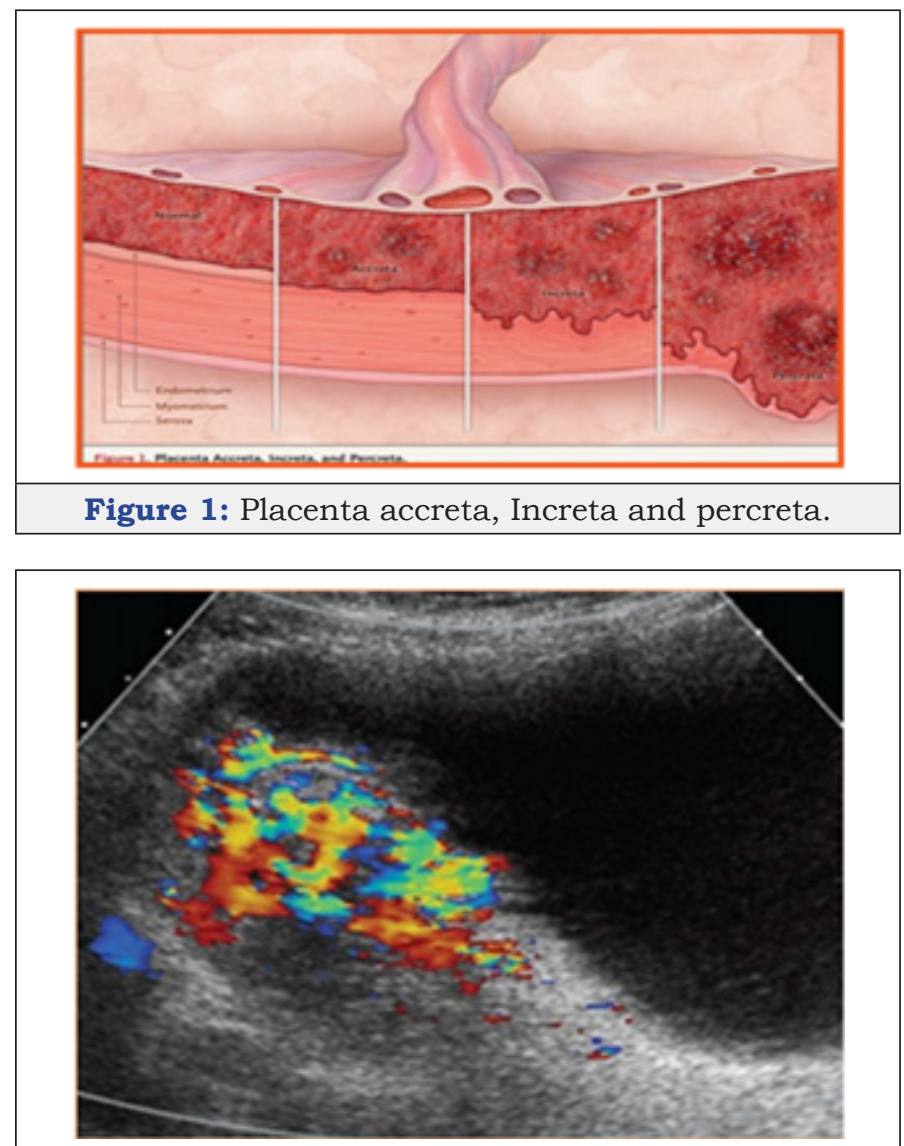

Figure 2: Turbulent flow in the gaps and increased vascularity between the placenta and bladder in a case of percrete placenta, using doppler color.

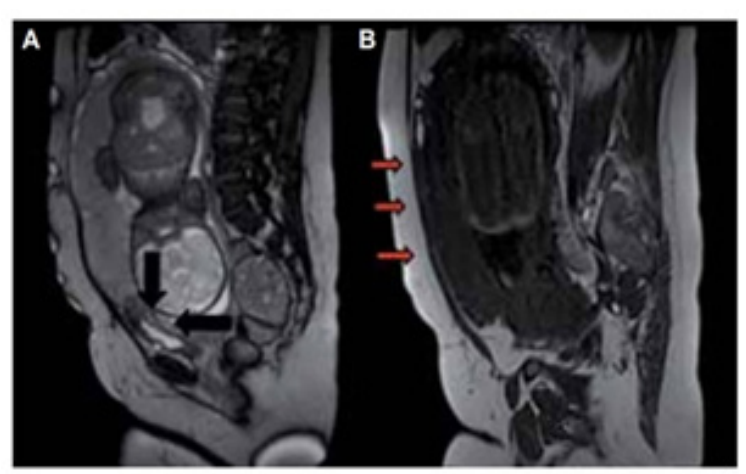

Figure 3: A Sagittal image, weighted at T2. It is noted irregularity of the bladder bed with loss of the interface separating the myometrium and the placenta. B. T1weighted sagittal image.

\section{Clinical Case}

38-year-old female, 6 deliveries 2 caesarean sections 3 without other important pathological history, who went to the emergency department at 33.1 weeks of gestation for presenting little transvaginal bleeding. On physical examination without active bleeding, ultrasound with fetometry according to gestational age, total occlusive placenta previa, loss of the uteroplacental interphase with penetrating vessels towards myometrium and presence of multiple gaps suggesting that bladder percretism be ruled out. Lung maturity scheme is started and graduated due to improvement. Patient cited 2 weeks later to resolve the pregnancy via the abdomen, undergoing a subarachnoid block, with a mid-infraumbilical incision and dissection through planes until reaching the abdominal cavity, once the uterus was located, bladder-penetrating vessels were identified, and a hysterotomy was decided. Transplacental transverse body type (Figure 4). A live male newborn with a weight of 2470 grams, Apgar 7/8 and Capurro of 36 weeks was extracted. It proceeds.

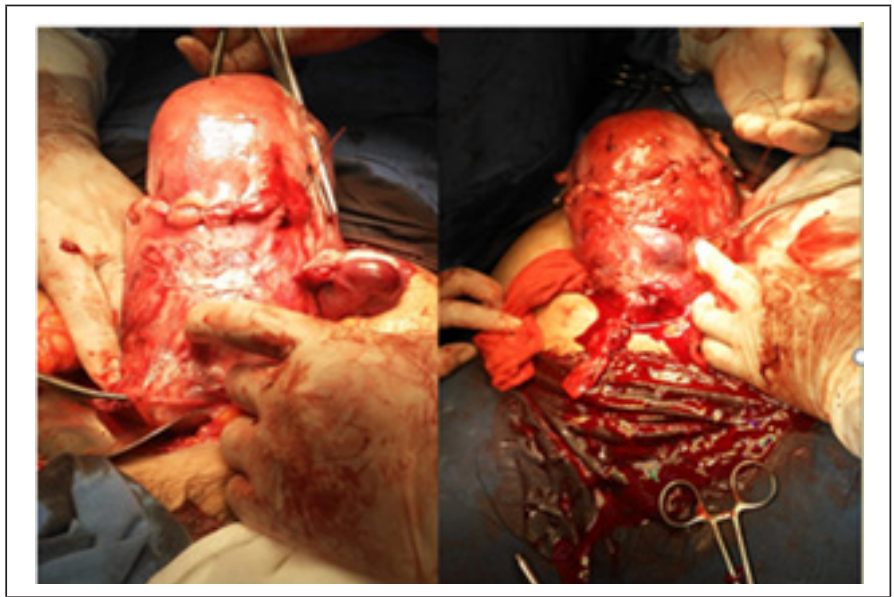

Figure 4: A. Uterine transplacental incision site. B. Uterovesical neoformation vessels.

Hysterorrhaphy in a plane with chromic catgut 1 and total intrafacial abdominal hysterectomy, dissecting the bladder gently and ligating neoformation vessels of greater caliber (Figure 4). The surgical piece is removed, and the cupola is left semi-open. Ligation of the hypogastric arteries is performed using the Gala technique. Before layer bleeding, packing is performed with placement of 2 penrose towards parietocolic slides. Mala bag is placed and the abdominal wall is closed with points of confrontation, total bleeding of 4000cc is admitted to the Obstetric Intensive Care Unit (UCIO), 48 hours later, unpacking is performed, repairing the bladder dome with imbrincate points, and proceeds with a closed defi-Abdominal wall tissue with estimated bleeding of 200cc with re-admission to the ICU, being extubated after 48 hours. It is delivered with a silicone foley catheter 6 days after the last surgical event. Appointed after 10 days without complications and by appointment at the urology outpatient clinic to perform cystoscopy and remove the foley catheter. 


\section{Discussion}

One of the most important modifiers of the clinical result is the prenatal identification of placental accretion, which allows optimal management, which generally includes a planned caesarean section hysterectomy before the start of labor or bleeding. Women with clinical or ultrasound risk factors for BP should be referred to a center of excellence or imaging expert for evaluation of the invasive placenta [5]. Magnetic resonance imaging is only recommended for cases in which the placenta cannot be seen adequately by ultrasound, such as in posterior placentation or in multiple pregnancies. Most experts recommend a cesarean section planned hysterectomy with fundic hysterotomy to avoid compromising the placenta. The entrance to the abdominal cavity is through a mid-infra umbilical abdominal incision, and the pregnant uterus is gently exteriorized to allow for fundic hysterotomy. Once this area is opened to the level of the amnion, the myometrium can be raised by the surgeon's fingers, which serves to plug the venous sinuses and protect the fetus, the incision can be extended using an electrocautionary.

The placenta is allowed to remain in situ. The hysterectomy is quickly closed with 1-0 Vicryl suture with anchored stitches. Total abdominal hysterectomy is performed to ensure margins around any placental tissue, and to isolate and manage any neovascularization to the uterus in a systematic manner. Several current publications refer to completing the surgical technique to have a greater field view as follows: access the lateral retroperitoneum to the round ligament, exposing and identifying the ureters and iliac vessels. The uterus is then separated from its support, leaving as wide a margin of broad ligament as possible. The ovaries are preserved, but the tubas are removed. This technique allows us to identify any additional feeder arteries (such as the superior bladder) to temporarily occlude them with a vascular clip, if necessary. Step by step, this devascularization is able to reduce the congestion of the lower segment and the bladder wall from the beginning. When necessary, ureterolysis is performed to protect the ureters and allow step-by-step devascularization of the lower segment. Even in cases of PA and PI, in which the trophoblast has not invaded the bladder wall, there may still be highly distended and congested blood vessels that pass through the interface between the bladder and the lower uterine segment that can cause significant bleeding. By exposing the anatomy of the lateral pelvis and the performance of a ureterolysis, these vessels can be cauterized and sealed, and the bladder can be separated in a controlled manner with minimal blood loss [5].

Incidental injury to the bladder is itself favored by the diagnosis of PP to the bladder, as well as by fibrosis of the adhesion to the anterior wall of the uterus with the consequent firm division of tissues. There are multiple surgical modifications of caesarean section hysterectomy, which can be used at present for the management of BP, such as the Esperanza Bautista techniques, Rodríguez Bosh technique or Jaraquemada technique, to mention a few, however, in our Hospital it highlights the technique of Dr. Malagón Reyes with which good surgical results have been obtained. The technique is as follows: A 5 Fr neonatal feeding tube is inserted into the umbilical vein and $2 \mathrm{cc}$ of sclerosing solution ( $3 \%$ polidocanol) are instilled with the tessari technique, later limiting uterine flow (ligation of uterine arteries and hypogastric arteries internal cas), a gentle dissection of the vesicouterine plication is performed, the usual hysterectomy technique is continued. This technique has considerably reduced maternal morbidity and mortality, from bleeding in our patients, to admission to the ICU due to hypovolemic shock [5].

\section{Conclusion}

Prenatal identification of placental accretion is crucial for both medical and surgical decision making. Due to the high morbidity and mortality rates associated with accretion, it is essential to have the multidisciplinary team that generates better diagnostic and therapeutic conditions for the patient. Knowing other surgical options will generate greater success in the maternal-fetal future of the binomial.

\section{References}

1. Silver RM, Branch DW (2018) Placenta acreta spectrum. N Engl J Med 378(16): 1529-1536.

2. Jauniaux E, Collins S, Burton GJ (2018) Placenta accreta spectrum: Pathophysiology and evidence-based anatomy for prenatal ultrasound imaging. Am J Obstet Gynecol 218(1): 75-87.

3. Silver RM (2015) Abnormal placentation: Placenta previa, vasa previa, and placenta accreta. Obstet Gynecol 126(3): 654-668.

4. Belfort MA, Shamshirsaz AA, Fox KA (2018) The diagnosis and management of morbidly adherent placenta. Semin Perinatol 42(1): 4958.

5. Malagón R, Ángeles M, Salgado I (2013) Images in the Obstetric Intensive Care Unit (UCIO). Journal of the Mexican Association of Critical Medicine and Intensive Care, XXVII, pp.119-121. 